\title{
Arsenic Trioxide Increases Apoptosis of SK-N-BE (2) Cells Partially by Inducing GPX4-mediated Ferroptosis
}

\section{Chuchu Feng}

Sun Yat-Sen Memorial Hospital https://orcid.org/0000-0002-4390-0119

\section{Yu Wu}

Sun Yat-Sen Memorial Hospital

\section{Yantao Chen}

Sun Yat-Sen Memorial Hospital

\section{Xilin Xiong}

Sun Yat-Sen Memorial Hospital

\section{Peng Li}

Guangzhou Institutes of Biomedicine and Health

\section{Xiaomin Peng}

Sun Yat-Sen Memorial Hospital

\section{Choumou Li}

Sun Yat-Sen Memorial Hospital

\section{Wenjun Weng}

Sun Yat-Sen Memorial Hospital

\section{Yafeng Zhu}

Sun Yat-Sen Memorial Hospital

\section{Dunhua Zhou}

Sun Yat-Sen Memorial Hospital

Yang Li ( $\nabla$ drliyang@126.com )

Sun Yat-Sen Memorial Hospital https://orcid.org/0000-0002-1756-5847

\section{Research Article}

Keywords: Neuroblastoma, Arsenic trioxide, Ferroptosis, GPX4, Quantitative proteomic analysis

Posted Date: February 9th, 2022

DOI: https://doi.org/10.21203/rs.3.rs-1271272/v1 
License: (c) (i) This work is licensed under a Creative Commons Attribution 4.0 International License. Read Full License 


\section{Abstract}

Neuroblastoma (NB) is the most common extracranial tumor in central nervous system threatening children's health with limited therapeutic options. Arsenic trioxide (ATO) has been identified the cytotoxicity in NB cells but the potential mechanism remains unclear. In this study, we attempted to obtain some insight into the mechanisms of cell death induced by ATO in NB cells using proteomic technology. Proteomic analyses found that ATO can affect the signaling pathway associated with ferroptosis, including the upregulation of iron absorption (FTL, FTH1, HO-1), ferritinophagy (LC3, P62, ATG7, NCOA4) and modifier of glutathione synthesis (GCLM); downregulation of glutamine synthetase (GS) and GPX4, which was the critical inhibitor of ferroptosis. Western blot analysis revealing GPX4 expression in SK-N-BE (2) cells decreased after treatment with ATO $(7.3 \mu \mathrm{M})$, resulting in a loss of GPX4 activity. Our study revealed that ATO may induce ferroptosis in neuroblastoma cell SK-N-BE (2) by facilitating the downregulation of GPX4, ultimately resulting in iron-dependent oxidative death.

\section{Introduction}

Quantitative proteomics is predominantly conducted using mass spectrometry (MS)-based technologies[1], where proteins are extracted and digested into peptides by a sequence-specific enzyme, and quantifiedby different quantification strategies such as label-free quantification (LFQ)[2].

Quantitative proteomics can identify novel protein as well as differentially expressed, which is a powerful technique for revealing the underlying mechanisms of cancer and therapeutic targets. Based on the advantages that serum/plasma has a vast array of proteins complexes and ease of serial sample collection, proteomics has been applied in detection, diagnosis, prognostic judgment, or management of cancers[3-5].

Neuroblastoma (NB)is the most common extracranial tumor in childhood with the 7-8\% incidence rate and approximately $15 \%$ mortality rate accounts for of all pediatric cancer deaths. Several recent reports highlight the potential use of a proteomics in investigating the pathobiology of NB. Murillo et al.[6] used iTRAQ-based proteomics to analyze potential differentiation markers which were similar to neuronal differentiation aspects, and select MARCKS, STMN1, AIFM1, SMN1, AGRN and CTNND1 to be used as candidates applied in mature NB phenotype. LFQ proteomic profiling revealed the proteins involved in cell communication and signal transduction in exosomes which released by the MYCN-amplified NB cells[7], suggesting that exosomes play an role in chemoresistance of MYCN-driven aggressive NB. In a study by Lorena et al.[8],protein expression patterns of distant metastases in NB xenograft mouse model were identified by Quantitative proteomics. Hwang et al.[9] found a total of 12 proteins differentially regulated after LGR5 knockdown, including Wnt/ $\beta$-catenin signaling and hnRNP family, indicating that LGR5 and its downstream signaling may play a critical role in NB by hyperactivation of alternative pre-mRNA processing.

According to clinical manifestation, biological characteristics and age, NB patients can be divided into very low risk, low risk, intermediate risk, or high risk, respectively. High-risk NB has a poor prognosis with 5 
years overall survival (OS) rate of less than $15 \%$ after the multimodality therapy[10] and exceed $80 \%$ recurrence rate within 2 years[11]. Therefore, there is an urgent need to identify novel drugs or therapeutic approaches for patients with High-risk NB. Proteome analysis has been used to identify the molecular mechanisms of potential drugs for NB. For example, Halakos et al.[12] found that cysteine protease inhibitor K777 combined with 13-cis RA could stimulate NB cell lines differentiation by multiple signaling pathways, whereas RET, GDF15, APP, ADAM10, and PLAT may be a novel biomarker of differentiation. Oliva et al.[13] found that upregulation of GLS2 in NB cell line SH-SY5Y induced an antiproliferative response with upregulation of tumor suppressor p53 and p21 protein and cell cycle arresting at the G2/M phase. AURKA and AURKB were significantly associated with MYCN-amplified and high-risk NB. Aurora kinase inhibitor tozasertib was found to suppress tumor cells migration and invasion ability in NB ThMYCN mouse model via regulated the actin cytoskeleton as well as altered fatty acid and carbohydrate metabolic processes[14]. Therefore, proteomic analysis can help in drug discovery, unraveling drug mechanisms of action and drug clinical application.

As early as the 1970s, Chinese scholars discovered that Arsenic Trioxide (ATO) can induce acute promyelocytic leukemia (APL) tumor cell differentiation and apoptosis [15]. Since then, dozens of studies showed the broad-spectrum anti-tumor activities of ATO[16-20]. One of the interesting findings was that ATO increasing the levels of malonaldehyde (MDA) and reactive oxygen species (ROS), while decreasing glutathione (GSH); The accumulation of ROS could trigger oxidative stress and damage mitochondrial membrane potential in spermatogonia [21]and pulmonary fibroblast cell [22], therefore activate downstream caspase-dependent apoptosis pathways[23],this allowed ATO to be inferred can treat cancer effectively through this mechanism. Our previous studies confirmed the anti-tumor growth effect of ATO in NB cells. ATO could inhibit the NB cells growth in vitro by means of arresting NB cells in G2/M phase[24], increasing TrkA/C receptors which related to the good prognosis of $N B[25]$ and reducing multidrug-resistance protein[26]. Our previous work [27]found that ATO can restore the sensitivity of chemotherapy in some relapsed/refractory NB patients. Preliminary data[28] of our ongoing clinical trial (ChiCTR1800014748, NCT03503864) also showed that ATO combined with chemotherapy can significantly improve induction response in 4/M neuroblastoma without serious adverse effects. To further improve the clinical therapeutic effect and explore the mechanisms underlying ATO treatment, in this study, we compared SK-N-BE (2) cells proteome before and after ATO treatment by using LFQ proteomic analysis. In view of the results issued from the proteomic analyses, complementary experiments were conducted to gain a deeper insight into the mechanisms of ATO killing NB cells.

\section{Results}

\section{ATO inhibit the proliferation of SK-N-BE (2) cells}

The neuroblastoma cell line SK-N-BE (2) were cultured with gradient concentration of ATO for 24hours. Consistent with our previous research[24, 25], the results revealed that ATO inhibited SK-N-BE(2) cells 
(Fig. 1). ATO inhibited the growth of SK-N-BE(2) cells in a concentration-dependent manner from $4 \mu \mathrm{M}$ to $16 \mu \mathrm{M}$ and the $\mathrm{IC}_{50}$ of ATO were $7.3 \mu \mathrm{M}$.

\section{Protein intensity information indicated before and after ATO incubation}

To compare the protein expression profiles from SK-N-BE (2) cells before and after ATO treatment, the mass spectrometry-based on liquid chromatography (LC-MS) was adopted for conducting the label-free proteome quantitation (LFQ)to identify protein intensities information. The ratio of LFQ between ATOtreated and negative control (NC) samples upon two times was considered to indicate a significant difference. After 24 hours of ATO $\left(\mathrm{IC}_{50} 7.3 \mu \mathrm{M}\right)$ incubation, we identified 6095 proteins where 291 and 386 proteins were significantly upregulated and downregulated in ATO-treated group, respectively. The proteins were classified by Gene Ontology (GO) annotation based on three categories: cellular component, molecular function and biological process. For each category, the enrichment of the differentially expressed protein against all identified proteins have detected (Fig. 2). Differentially expressed proteins were mainly composed of nucleus (38\%), cytoplasm (27\%), extracellular (10\%), mitochondria (8\%) and plasma membrane (8\%). Molecular functions include binding (55\%), catalytic activity (18\%) and molecular function regulators (11\%). Differential proteins were involved in the cellular processes (17\%), biological regulation (13\%), single-organism process (13\%), response to stimulus (10\%) and metabolic process (9\%).

\section{Signaling pathway regulated by ATO in SK-N-BE (2) cell}

To further know information on molecular interaction networks, Encyclopedia of Genes and Genomes (KEGG) database was used to identify enriched pathways which were classified into hierarchical categories according to the KEGG website (http://www.kegg.jp/kegg/mapper.html). In accordance with the enrichment of pathway analysis, we identified pathways in mineral absorption, ferroptosis and autophagy as significant biological processes in SK-N-BE(2) cells after ATO treatment(Fig. 3).Proteins differentially upregulated were ferritin light (FTL), heavy chain (FTH1), Heme oxygenase (HO)-1 and glutamate cysteine ligase (GCL)in mineral absorption and ferroptosis, as well as autophagy related proteins microtubule-associated proteins 1A/1B light chain 3 beta (LC3/MAP1LC3B), autophagy protein (ATG7), nuclear receptor coactivator 4 (NCOA4) and P62. On the contrary, the expression of GS and GPX4 were significantly reduced (Fig. 4).

\section{Effects of ATO on the GPX4 in SK-N-BE (2) cell}

As shown in Fig. 5, western blotting revealed that GPX4 expression was profoundly decreased under 7.3 $\mu \mathrm{M}\left(\mathrm{IC}_{50}\right)$ ATO treatment for 24 hours. The enzyme activity of GPX4 were downregulated in $9 \mu \mathrm{M}$ ATO treatment more notably than in control group without ATO (Fig. 6A). It was also observed that GSH appeared decreased in 9 and $12 \mu \mathrm{M}$ ATO treatment in comparison to $3 \mu \mathrm{M}$ group (Fig. 6B).

\section{Discussion}


As one of the oldest drugs, ATO has shown efficacy against hematologic malignancies and various solid tumors. Previous studies have shown that ATO inhibited NB cells growth via activation of apoptosisrelated proteins[29]. Lowest dose of ATO could induce the oxidative stress and cause the alterations of BCL2, BID and BCL-x/L, and activation of caspase-3 and-9, resulting in activation of apoptotic signaling pathways[30]. However, the detailed molecular mechanisms of ATO action are is still largely unknown. To identify novel mechanism of ATO action in NB cells, we investigated the changes in the proteome after ATO treatment in SK-N-BE (2) cells. It had been reported that ATO induced ROS production[21], and in the present study we identified a group of proteins related to ferroptosis and ferritinophagy, such as GPX4, ferritin (FTL, FTH1), LC3, P62, ATG7 and NCOA4.

Ferroptosis is a form of regulated cell death which is distinct from other identified types of cell death such as apoptosis and necrosis. It is characterized by the accumulation of lipid ROS derived from iron overload, first demonstrated by Stockwell et al. in 2012[31, 32]. In general, the ferroptosis is mainly induced by lipid peroxidation, which is triggered by inactivation of the GPX4 because of the glutathione (GSH) exhaust, or by iron overload leading to ROS production[33].GPX4 activity is essential to maintain lipid homeostasis in the cell, prevent the accumulation of toxic lipid peroxides and thereby block the onset of Fenton chemistry catalyzed by intracellular iron, preventing ferroptosis from happening[34]. Our proteomic results showed that GPX4 protein expression in ATO group decreased significantly, and western blotting findings were consistent with proteomic analysis. Moreover, at higher concentrations of ATO, the amount of intracellular GSH dropped, meanwhile, a decrease in the enzyme activity of GPX4 was noticed. Glutamine (Gln) is a nonessential amino acid with the roles in tricarboxylic acid (TCA) cycle, promoting cell proliferation as a carbon and nitrogen sources of the tumor. Glutamine synthetase (GS) metabolizes glutamate (Glu) to Gln while Glu is synthesized from Gln by glutaminase (GLS)[35, 36]. GSH consists of Glu, cysteine and glycine, and it is synthesized by the rate limiting enzymes glutamyl cysteine ligase (GCL). GCL is composed of two subunits, catalytic (GCLC) and modifier (GCLM)[37]. Our proteomic results showed that the expression of GS protein was significantly decreased while the GCLM upregulated after ATO exposure; in the meanwhile, reduced GSH and GPX4 activity were found to be significantly low, speculating that ATO may reduce the intracellular GIn synthesis and dampen down intracellular GSH, finally resulting in the inhibition of GPX4 activity.

Proteomic data also showed that there was significant variation in ferritin (FTL, FTH1) proteins levels upon ATO exposure, following the increase in $\mathrm{HO}-1$ protein[38]. The heme oxygenase $(\mathrm{HO})$ is a major intracellular source of iron through detoxifieing heme into biliverdin, releasing carbon monoxide and $\mathrm{Fe}^{2+}$ [39]. It has been reported that erastin induces HO-1-dependent ferroptotic cell death in fibrosarcoma cells by take part in iron supplement and lipid peroxidation[40,41]. The process of autophagosomes degradate ferritin and release free iron called as "ferritinophagy"[42-44]; Lysosomal iron leading to lysosomal bursting and cell death via Fenton-like reactions [45]. Indeed, the significant up-expression of LC3, P62, ATG7 and NCOA4 indicated the autophagy initiation after ATO exposure. NCOA4 was highly enriched in autophagosomes, revealing ferritin heavy and light chains and mediating the transport of ferritin to the 
lysosome via the autophagosome. Our results suggested that ferritinophagy may be involved in ATOinduced ferroptosis, but further experimental validation is needed to validate this.

In recent researches, ferroptosis-inducing agents could be developed as cancer therapy strategies: Sorafenib was found as an inducer of ferroptosis in hepatocellular carcinoma (HCC) cell lines and its cytotoxic effect could be prevented by iron chelation[46]. Sulfasalazine, used to anti-inflammatory, could induces ferroptotic cell death in glioma cells[47]. Antimalarial drug artesunate been reported can selectively killed HNC cells by generate ROS and lead to oxidative stress in cancer cells[48].N Genget al. identified that the oncogenic RAS-selective lethal small molecule erastin induced ferroptosis in NB cells and decreased the expression of Fpn gene and protein. Fpn siRNA transfection in NB cells could enhance anticancer activity of erastinby increasing iron-dependent lipid ROS accumulation[49].It also reported that arsenic induced ferroptotic cell death in neuron of mouse model by accumulation of reactive oxygen species and lipid peroxidation products, disruption of $\mathrm{Fe}^{2+}$ homeostasis, this is consistent with our in vitro experimental results [50]. Our study further confirmed a decrease in the levels of GPX4 protein in parallel with the loss of its enzyme activity after ATO exposure. The significant finding of our study is that ferroptosis activated by ATO may contribute to the major form of SK-N-BE (2) cell death. Expression levels of key proteins in the ferroptotic molecular pathway were up-regulated in response to ATO exposure. Moreover, ATO-induced ferroptosis was probably in a manner by ferritinophagy involving increasing iron accumulation.

\section{Conclusion}

Here, we found ATO may as a ferroptosis-inducing agent in neuroblastoma, which may act through the suppression of GPX4 protein and activation of ferritinophagy. Further research is needed to confirm our conjecture.

\section{Materials And Methods}

\section{Cell lines and culture}

Human SK-N-BE (2) cells were obtained from the Sun Yat-sen University (Guangzhou, China). SK-N-BE (2) cells were cultured in DMEM medium supplemented with $10 \% \mathrm{FBS}, 100 \mu \mathrm{g} / \mathrm{ml}$ penicillin, and $100 \mu \mathrm{g} / \mathrm{ml}$ streptomycin at $37^{\circ} \mathrm{C}$ and $5 \% \mathrm{CO}_{2}$. Subsequent to growing in a monolayer and multilayers with $80 \%$ confluency, the cells were transferred to 96 -well plates at a density of $1 \times 10^{4}$ cells or 6 -well plates at a density of $1 \times 10^{5}$ cells. The ATO was produced by Harbin Medical University Pharmaceutical Company (Harbin, China).

\section{Cytotoxicity assays}

Growth inhibition of SK-N-BE (2) cells was assessed using Cell Counting Kit-8 (CCK-8) (APExBIO, K1018). For the CCK-8 assay, a total of $1 \times 10^{4}$ cells were seeded in 96 -well microculture plates and treated with 
ATO $\left(1,2^{1} \bowtie 2^{7} \mu \mathrm{M}\right)$ for 24 hours. Then, $10 \mu \mathrm{l} / 100 \mu$ l of the CCK-8 solution were pipetted into each well. After incubating for 1 hours, the absorbance of plate was measured at $450 \mathrm{~nm}$ using plate reader Molecular Devices, LLC, Sunnyvale, CA, USA) to calculate percentages of survival cells. The cell proliferation rate was calculated as the percentage of CCK-8 absorption as follows: [(As-Ab)/(Ac-Ab)]×100\%. As: Absorbance of drug treated sample. Ab: absorbance of blank control sample (without cells). Ac: absorbance of control sample.

\section{Label-Free Comparative Proteomics Analysis}

\section{Protein Digestion and TMT Labeling}

Sample was sonicated three times in lysis buffer and centrifuged at $12000 \mathrm{~g}$ for $10 \mathrm{~min}$ at $4^{\circ} \mathrm{C}$. The protein concentration of supernatant was determined with BCA kit. Then, the protein solution was reduced and alkylated. The trypsin was added at 1:50 trypsin-to-protein mass ratio for the first digestion overnight and 1:100 trypsin-to-protein mass ratio for a second $4 \mathrm{~h}$ digestion. Digested peptides were then dried and reconstituted with 0.5 M TEAB. Finally, the sample were labeled according to the manufacturer's protocol for TMT kit/iTRAQ kit.

\section{LC-MS/MS Analysis}

As for separation of peptide fragment by EASY-nLC 1000 UPLC, the tryptic peptides were dissolved in $0.1 \%$ formic acid (solvent $A$ ), then separated on the analytical column with a gradient elution at a constant flow rate of $400 \mathrm{~nL} / \mathrm{min}$. The gradient was comprised of an increase from $6-23 \%$ solvent $\mathrm{B}$ ( $0.1 \%$ formic acid in $98 \%$ acetonitrile) over $26 \mathrm{~min}, 23-35 \%$ in $8 \mathrm{~min}$ and climbing to $80 \%$ in $3 \mathrm{~min}$ then holding at $80 \%$ for the last $3 \mathrm{~min}$. The eluted peptides were subjected to a nanospray ionization (NSI) source followed by tandem mass spectrometry (MS/MS) in Q ExactiveTM Plus coupled online to the ultra-performance liquid chromatography (UPLC) with $2.0 \mathrm{kV}$ electrospray voltage. Resolution was 17,500 at $350-1800 \mathrm{~m} / \mathrm{z}$ for tandem mass spectrometry scans. A data-dependent procedure that alternated between one MS scan followed by $20 \mathrm{MS} / \mathrm{MS}$ scans with 15.0 s dynamic exclusion. Automatic gain control (AGC) was set at $5 E 4$. Fixed first mass was set as $100 \mathrm{~m} / \mathrm{z}$. The resulting MS/MS data were processed using Maxquant search engine (v.1.5.2.8). Tandem mass spectra were searched against human uniprot database concatenated with reverse decoy database.

\section{GPX4 activity assay and Gluthione determination}

The activity of GPX4 was determined using a Glutathione Peroxidase Assay Kit (KeyGEN BioTECH). Intracellular reduced form gluthione (GSH) were assessed using a GSH Detection Assay Kit II (KeyGEN BioTECH) according to the manufacturer's instructions.

\section{Western blot analysis to investigate the expression of GPX4}

After treatment with appropriate drug concentration, protein lysate was prepared in lysis buffer and the protein concentration was determined by BCA protein assay. SDS-PAGE gel, and electro-transferred to 
PVDF membrane. The membrane was washed in TBST and blocked with $5 \%$ nonfat dry milk in TBST. Membranes were incubated overnight at $4^{\circ} \mathrm{C}$ with GPX4 primary antibody (1:1000). Peroxidaseconjugated secondary antibody (1:1000) was used as the secondary antibody. After electrophoresis, proteins were transferred to a nitrocellulose membrane and probed by corresponding antibodies.

\section{Declarations}

\section{Acknowledgements}

This work was supported by Grant 2017A030313806 and 2020A1515010127 from the Natural Science Foundation of Guangdong Province; Grant SYS-C-202007 form Sun Yat-Sen Clinical Research Cultivating Program.

\section{Conflict of interest}

The authors declare that there is no potential competing interest.

\section{Ethical approval}

This article does not contain any studies with human participants or animals performed by any of the authors.

\section{References}

1. Aebersold R, Mann M: Mass-spectrometric exploration of proteome structure and function. Nature 2016, 537(7620):347-355.

2. Ankney JA, Muneer A, Chen X: Relative and Absolute Quantitation in Mass Spectrometry-Based Proteomics. Annu Rev Anal Chem (Palo Alto Calif) 2018, 11(1):49-77.

3. Larkin SE, Johnston HE, Jackson TR, Jamieson DG, Roumeliotis TI, Mockridge Cl, Michael A, Manousopoulou A, Papachristou EK, Brown MD et al: Detection of candidate biomarkers of prostate cancer progression in serum: a depletion-free 3D LC/MS quantitative proteomics pilot study. $\mathrm{Br} J$ Cancer 2016, 115(9):1078-1086.

4. Timms JF, Arslan-Low E, Kabir M, Worthington J, Camuzeaux S, Sinclair J, Szaub J, Afrough B, Podust VN, Fourkala EO et al: Discovery of serum biomarkers of ovarian cancer using complementary proteomic profiling strategies. Proteomics Clin App/ 2014, 8(11-12):982-993.

5. Sung HJ, Cho JY: Biomarkers for the lung cancer diagnosis and their advances in proteomics. $B M B$ Rep 2008, 41(9):615-625.

6. Murillo JR, Goto-Silva L, Sanchez A, Nogueira FCS, Domont GB, Junqueira M: Quantitative proteomic analysis identifies proteins and pathways related to neuronal development in differentiated SH-SY5Y neuroblastoma cells. EuPA Open Proteom 2017, 16:1-11. 
7. Fonseka P, Liem M, Ozcitti C, Adda CG, Ang CS, Mathivanan S: Exosomes from N-Myc amplified neuroblastoma cells induce migration and confer chemoresistance to non-N-Myc amplified cells: implications of intra-tumour heterogeneity. J Extracell Vesicles 2019, 8(1):1597614.

8. Hanel L, Gosau T, Maar H, Valentiner U, Schumacher U, Riecken K, Windhorst S, Hansen NO, Heikaus L, Wurlitzer $\mathrm{M}$ et al: Differential Proteome Analysis of Human Neuroblastoma Xenograft Primary Tumors and Matched Spontaneous Distant Metastases. Sci Rep 2018, 8(1):13986.

9. Hwang M, Han MH, Park HH, Choi H, Lee KY, Lee YJ, Kim JM, Cheong JH, Ryu JI, Min KW et al: LGR5 and Downstream Intracellular Signaling Proteins Play Critical Roles in the Cell Proliferation of Neuroblastoma, Meningioma and Pituitary Adenoma. Exp Neurobiol 2019, 28(5):628-641.

10. Smith V, Foster J: High-Risk Neuroblastoma Treatment Review. Children (Basel) 2018, 5(9).

11. Basta NO, Halliday GC, Makin G, Birch J, Feltbower R, Bown N, Elliott M, Moreno L, Barone G, Pearson $A D$ et al: Factors associated with recurrence and survival length following relapse in patients with neuroblastoma. Br J Cancer 2016, 115(9):1048-1057.

12. Halakos EG, Connell AJ, Glazewski L, Wei S, Mason RW: Bottom up proteomics identifies neuronal differentiation pathway networks activated by cathepsin inhibition treatment in neuroblastoma cells that are enhanced by concurrent 13-cis retinoic acid treatment. $J$ Proteomics 2021, 232:104068.

13. Lopez de la Oliva AR, Campos-Sandoval JA, Gomez-Garcia MC, Cardona C, Martin-Rufian M, Sialana FJ, Castilla L, Bae N, Lobo C, Penalver A et al: Nuclear Translocation of Glutaminase GLS2 in Human Cancer Cells Associates with Proliferation Arrest and Differentiation. Sci Rep 2020, 10(1):2259.

14. Hsieh CH, Cheung CHY, Liu YL, Hou CL, Hsu CL, Huang CT, Yang TS, Chen SF, Chen CN, Hsu WM et al: Quantitative Proteomics of Th-MYCN Transgenic Mice Reveals Aurora Kinase Inhibitor Altered Metabolic Pathways and Enhanced ACADM To Suppress Neuroblastoma Progression. J Proteome Res 2019, 18(11):3850-3866.

15. Iland HJ, Bradstock K, Supple SG, Catalano A, Collins M, Hertzberg M, Browett P, Grigg A, Firkin F, Hugman $A$ et al: All-trans-retinoic acid, idarubicin, and IV arsenic trioxide as initial therapy in acute promyelocytic leukemia (APML4). Blood 2012, 120(8):1570-1580; quiz 1752.

16. Emadi A, Gore SD: Arsenic trioxide - An old drug rediscovered. Blood Rev 2010, 24(4-5):191-199.

17. Roboz GJ, Ritchie EK, Curcio T, Provenzano J, Carlin R, Samuel M, Wittenberg B, Mazumdar M, Christos PJ, Mathew $S$ et al: Arsenic trioxide and low-dose cytarabine in older patients with untreated acute myeloid leukemia, excluding acute promyelocytic leukemia. Cancer 2008, 113(9):2504-2511.

18. Lin CC, Hsu C, Hsu CH, Hsu WL, Cheng AL, Yang CH: Arsenic trioxide in patients with hepatocellular carcinoma: a phase II trial. Invest New Drugs 2007, 25(1):77-84.

19. Wei W, Zhou F, Zhang Y, Guo L, Shi H, Hou J: A combination of thalidomide and arsenic trioxide is effective and well tolerated in patients with myelodysplastic syndromes. Leuk Res 2012, 36(6):715719.

20. Grimm SA, Marymont M, Chandler JP, Muro K, Newman SB, Levy RM, Jovanovic B, McCarthy K, Raizer JJ: Phase I study of arsenic trioxide and temozolomide in combination with radiation therapy in patients with malignant gliomas. $J$ Neurooncol 2012, 110(2):237-243. 
21. Chen H, Liu G, Qiao N, Kang Z, Hu L, Liao J, Yang F, Pang C, Liu B, Zeng Q et al: Toxic effects of arsenic trioxide on spermatogonia are associated with oxidative stress, mitochondrial dysfunction, autophagy and metabolomic alterations. Ecotoxicol Environ Saf 2020, 190:110063.

22. You BR, Park WH: Arsenic trioxide induces human pulmonary fibroblast cell death via increasing ROS levels and GSH depletion. Oncol Rep 2012, 28(2):749-757.

23. Gupta S, Yel L, Kim D, Kim C, Chiplunkar S, Gollapudi S: Arsenic trioxide induces apoptosis in peripheral blood T lymphocyte subsets by inducing oxidative stress: a role of Bcl-2. Mol Cancer Ther 2003, 2(8):711-719.

24. Qi K, Li Y, Huang K, Xiong X, Chuchu F, Zhang C, Weng W: Pre-application of arsenic trioxide may potentiate cytotoxic effects of vinorelbine/docetaxel on neuroblastoma SK-N-SH cells. Biomed Pharmacother 2019, 113:108665.

25. Xiong X, Li Y, Liu L, Qi K, Zhang C, Chen Y, Fang J: Arsenic trioxide induces cell cycle arrest and affects Trk receptor expression in human neuroblastoma SK-N-SH cells. Biol Res 2018, 51(1):18.

26. Liu L, Li Y, Xiong X, Qi K, Zhang C, Fang J, Guo H: Low dose of arsenic trioxide inhibits multidrug resistant-related P-glycoprotein expression in human neuroblastoma cell line. Int J Oncol 2016, 49(6):2319-2330.

27. Li Y, Feng C, Chen Y, Huang K, Li C, Xiong X, Li P, Zhou D, Peng X, Weng W et al: Improved Outcomes with Induction Chemotherapy Combined with Arsenic Trioxide in Stage 4 Neuroblastoma: A Case Series. Technol Cancer Res Treat 2021, 20:15330338211041454.

28. Li C, Peng X, Feng C, Xiong X, Li J, Liao N, Yang Z, Liu A, Wu P, Liang X et al: Excellent Early Outcomes of Combined Chemotherapy With Arsenic Trioxide for Stage 4/M Neuroblastoma in Children: A Multicenter Nonrandomized Controlled Trial. Oncol Res 2021, 28(7):791-800.

29. Keim A, Rossler OG, Rothhaar TL, Thiel G: Arsenite-induced apoptosis of human neuroblastoma cells requires p53 but occurs independently of c-Jun. Neuroscience 2012, 206:25-38.

30. Yen CM, Tsai CW, Chang WS, Yang YC, Hung YW, Lee HT, Shen CC, Sheu ML, Wang JY, Gong CL et al: Novel Combination of Arsenic Trioxide (As203) Plus Resveratrol in Inducing Programmed Cell Death of Human Neuroblastoma SK-N-SH Cells. Cancer Genomics Proteomics 2018, 15(6):453-460.

31. Dixon SJ, Lemberg KM, Lamprecht MR, Skouta R, Zaitsev EM, Gleason CE, Patel DN, Bauer AJ, Cantley AM, Yang WS et al: Ferroptosis: an iron-dependent form of nonapoptotic cell death. Cell 2012, 149(5):1060-1072.

32. Kagan VE, Tyurina YY, Sun WY, Vlasova, II, Dar H, Tyurin VA, Amoscato AA, Mallampalli R, van der Wel PCA, He RR et al: Redox phospholipidomics of enzymatically generated oxygenated phospholipids as specific signals of programmed cell death. Free Radic Biol Med 2020, 147:231-241.

33. Chen X, Li J, Kang R, Klionsky DJ, Tang D: Ferroptosis: machinery and regulation. Autophagy 2020:128.

34. Forcina GC, Dixon SJ: GPX4 at the Crossroads of Lipid Homeostasis and Ferroptosis. Proteomics 2019, 19(18):e1800311. 
35. Bott AJ, Maimouni S, Zong WX: The Pleiotropic Effects of Glutamine Metabolism in Cancer. Cancers (Basel) 2019, 11(6).

36. Zhang J, Pavlova NN, Thompson CB: Cancer cell metabolism: the essential role of the nonessential amino acid, glutamine. EMBO J 2017, 36(10):1302-1315.

37. Yang Y, Dieter MZ, Chen Y, Shertzer HG, Nebert DW, Dalton TP: Initial characterization of the glutamate-cysteine ligase modifier subunit Gclm(-/-) knockout mouse. Novel model system for a severely compromised oxidative stress response. J Biol Chem 2002, 277(51):49446-49452.

38. Gozzelino R, Soares MP: Coupling heme and iron metabolism via ferritin $\mathrm{H}$ chain. Antioxid Redox Signal 2014, 20(11):1754-1769.

39. Loboda A, Damulewicz M, Pyza E, Jozkowicz A, Dulak J: Role of Nrf2/HO-1 system in development, oxidative stress response and diseases: an evolutionarily conserved mechanism. Cell Mol Life Sci 2016, 73(17):3221-3247.

40. Kwon MY, Park E, Lee SJ, Chung SW: Heme oxygenase-1 accelerates erastin-induced ferroptotic cell death. Oncotarget 2015, 6(27):24393-24403.

41. Hassannia B, Wiernicki B, Ingold I, Qu F, Van Herck S, Tyurina YY, Bayir H, Abhari BA, Angeli JPF, Choi $\mathrm{SM}$ et al: Nano-targeted induction of dual ferroptotic mechanisms eradicates high-risk neuroblastoma. J Clin Invest 2018, 128(8):3341-3355.

42. Asano T, Komatsu M, Yamaguchi-Iwai Y, Ishikawa F, Mizushima N, Iwai K: Distinct mechanisms of ferritin delivery to lysosomes in iron-depleted and iron-replete cells. Mol Cell Bio/ 2011, 31(10):20402052.

43. Kidane TZ, Sauble E, Linder MC: Release of iron from ferritin requires lysosomal activity. Am J Physiol Cell Physiol 2006, 291(3):C445-455.

44. Mancias JD, Wang X, Gygi SP, Harper JW, Kimmelman AC: Quantitative proteomics identifies NCOA4 as the cargo receptor mediating ferritinophagy. Nature 2014, 509(7498):105-109.

45. Kurz T, Gustafsson B, Brunk UT: Intralysosomal iron chelation protects against oxidative stressinduced cellular damage. FEBS J 2006, 273(13):3106-3117.

46. Lachaier E, Louandre C, Godin C, Saidak Z, Baert M, Diouf M, Chauffert B, Galmiche A: Sorafenib induces ferroptosis in human cancer cell lines originating from different solid tumors. Anticancer Res 2014, 34(11):6417-6422.

47. Sehm T, Fan Z, Ghoochani A, Rauh M, Engelhorn T, Minakaki G, Dorfler A, Klucken J, Buchfelder M, Eyupoglu IY et al: Sulfasalazine impacts on ferroptotic cell death and alleviates the tumor microenvironment and glioma-induced brain edema. Oncotarget 2016, 7(24):36021-36033.

48. Roh JL, Kim EH, Jang H, Shin D: Nrf2 inhibition reverses the resistance of cisplatin-resistant head and neck cancer cells to artesunate-induced ferroptosis. Redox Biol 2017, 11:254-262.

49. Geng N, Shi BJ, Li SL, Zhong ZY, Li YC, Xua WL, Zhou H, Cai JH: Knockdown of ferroportin accelerates erastin-induced ferroptosis in neuroblastoma cells. Eur Rev Med Pharmacol Sci 2018, 22(12):3826-3836. 
50. Tang Q, Bai L, Zou Z, Meng P, Xia Y, Cheng S, Mu S, Zhou J, Wang X, Qin X et al: Ferroptosis is newly characterized form of neuronal cell death in response to arsenite exposure. Neurotoxicology 2018, 67:27-36.

\section{Figures}

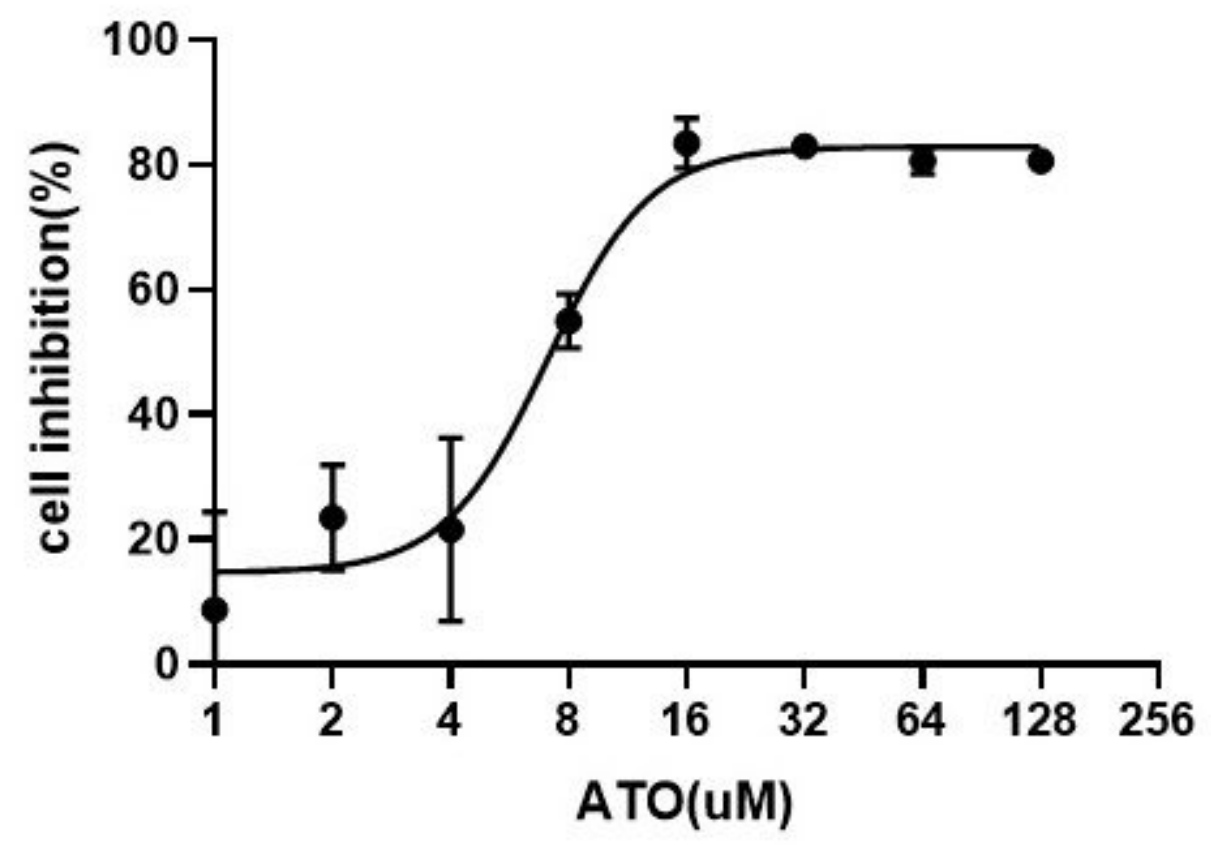

Figure 1

Inhibitory effects of ATO on SK-N-BE(2) cells proliferation.SK-N-BE(2) cells were treated with the indicated concentrations of $\operatorname{ATO}\left(1,2^{1} \otimes 2^{7} \mu \mathrm{M}\right)$ for 24 hours.

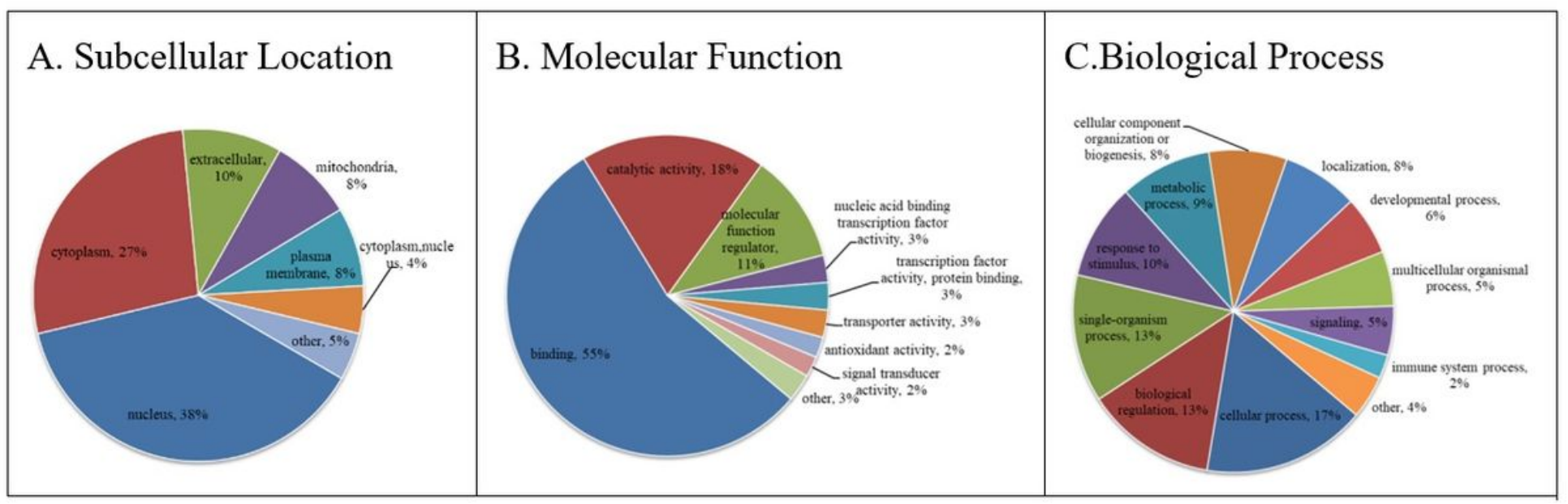

Figure 2 
Enrichment of Gene Ontology analysis. Proteins were classified by GO annotation into three categories: biological process, cellular compartment and molecular function.

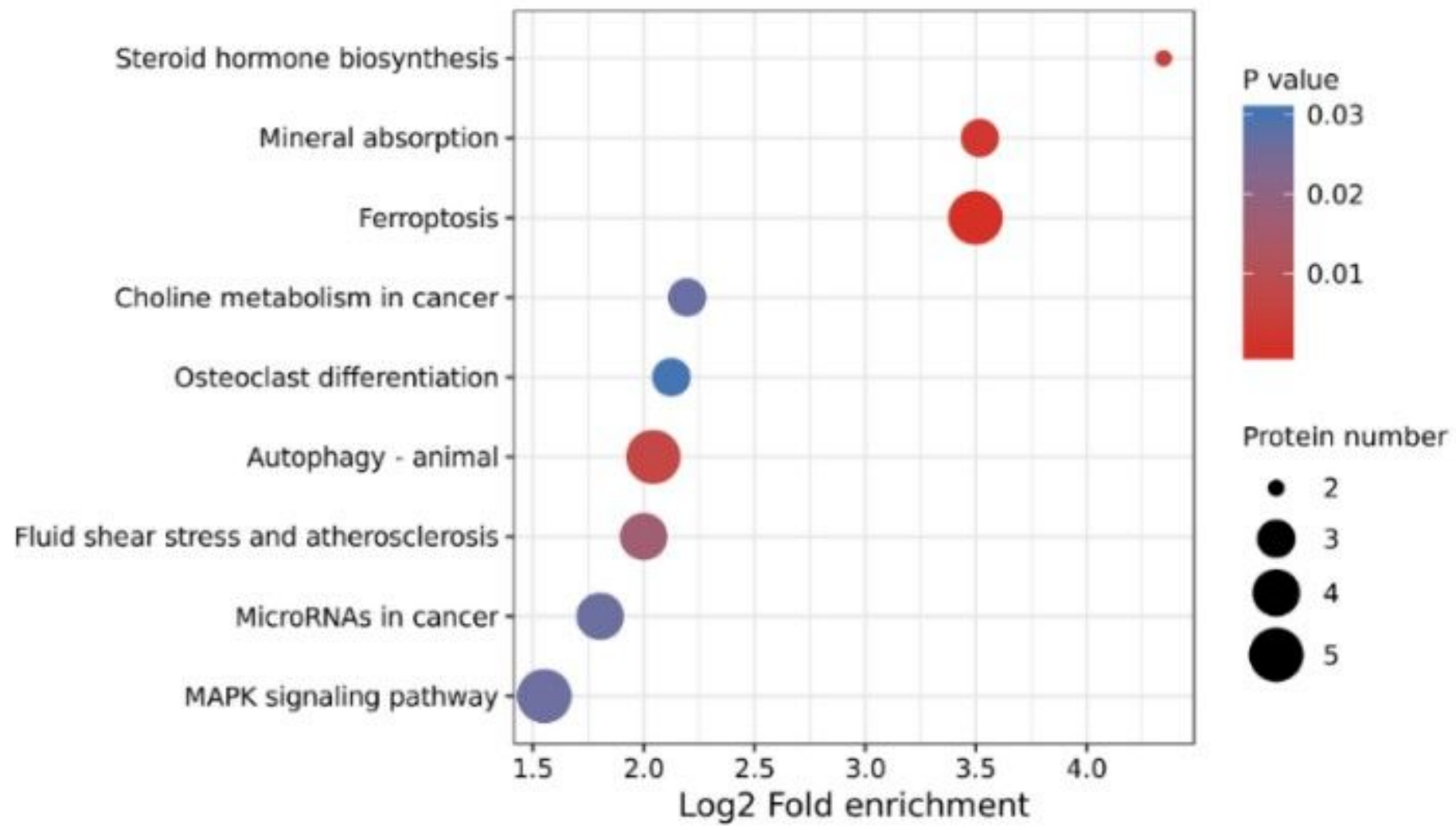

\section{Figure 3}

The pathway network regulated by ATO in SK-N-BE (2) cell. KEGG database was used to identify enriched pathways by a two-tailed Fisher's exact test to test the enrichment of the differentially expressed protein against all identified proteins. The pathway with a corrected $p$-value $<0.05$ was considered significant. 


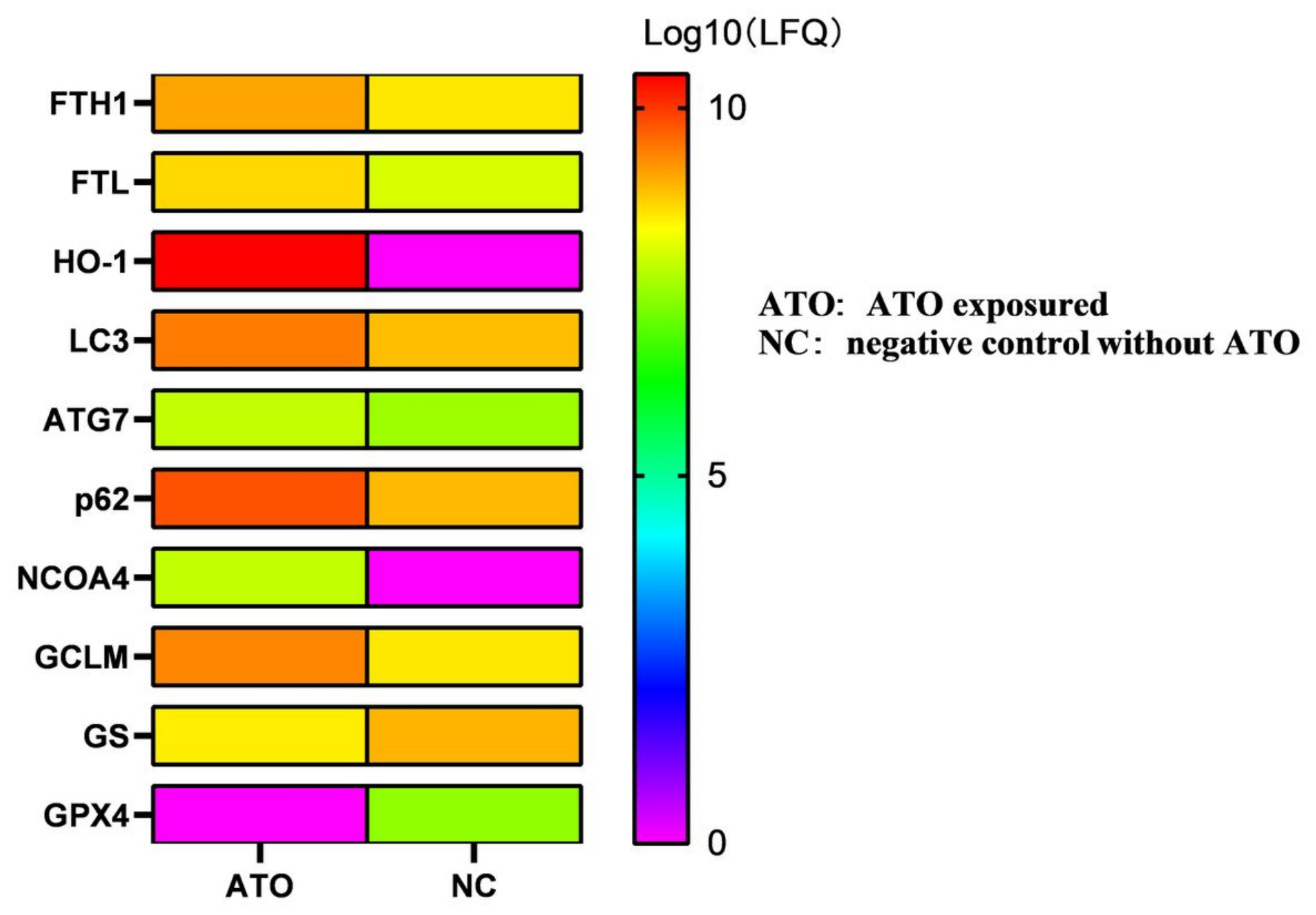

Figure 4

Differential protein expression of SK-N-BE (2) in mineral absorption, ferroptosis and autophagy pathways before and after ATO treatment.

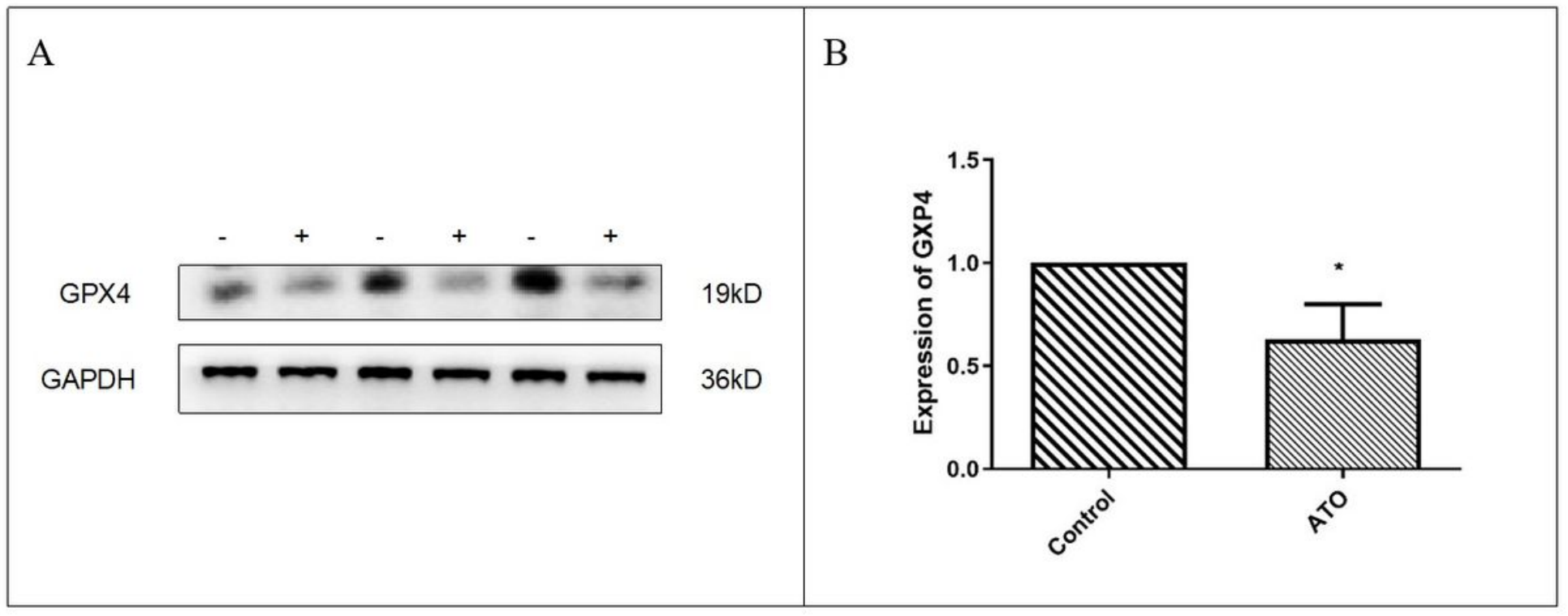


Figure 5

GPX4 expression is decreased by incubating the SK-N-BE (2) cells in the presence of ATO $(7.3 \mu \mathrm{M})$. ${ }^{*} \mathrm{P}<0.05$, compared with 24 hours control.

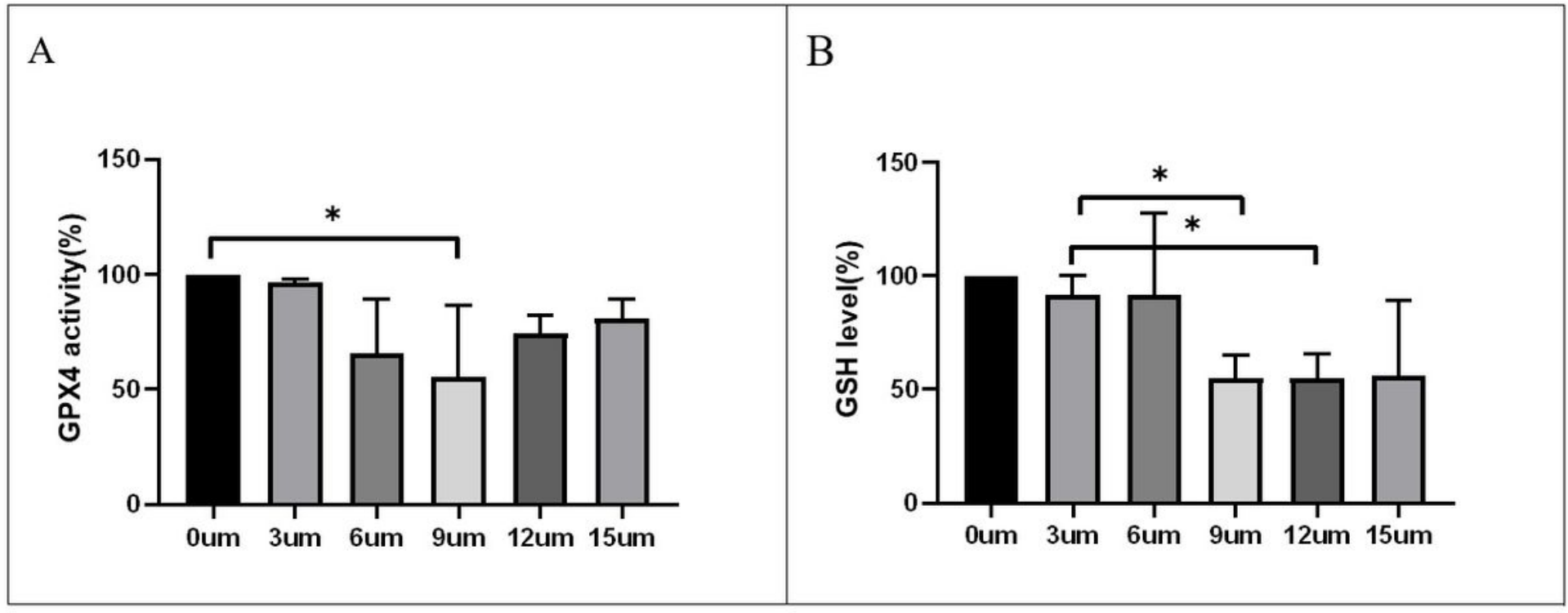

Figure 6

SK-N-BE (2) cells were treated with various concentrations of ATO for 24 hours. (A) The enzyme activity of GPX4 were downregulated in $9 \mu \mathrm{M}$ ATO. (B) The level of GSH decreased in high concentrations of ATO. 